\title{
Radiative Neutrino Mass, Dark Matter and Leptogenesis
}

\author{
Pei-Hong $\mathrm{Gu}^{1}$ * and Utpal Sarkar对 \\ ${ }^{1}$ The Abdus Salam International Centre for Theoretical Physics, Strada Costiera 11, 34014 Trieste, Italy \\ ${ }^{2}$ Physical Research Laboratory, Ahmedabad 380009, India
}

\begin{abstract}
We propose an extension of the standard model, in which neutrinos are Dirac particles and their tiny masses originate from a one-loop radiative diagram. The new fields required by the neutrino mass-generation also accommodate the explanation for the matter-antimatter asymmetry and dark matter in the universe.
\end{abstract}

PACS numbers: 14.60.Pq, 95.35.+d, 98.80.Cq

Introduction: Various neutrino oscillation experiments [1] have confirmed that neutrinos have tiny but nonzero masses. This phenomenon is naturally explained by the seesaw mechanism [2]. In the original seesaw scenario neutrinos are assumed to be Majorana particles, whose existence has not been experimentally verified so far. As an alternative, Dirac seesaw was proposed [3, 4] where the neutrinos can naturally acquire small Dirac masses. In the seesaw models, the observed matter-antimatter asymmetry in the universe can also be generated through the leptogenesis [5, 6, 7, 8, 9, 10]. Another big challenge to the standard model (SM) is the nature of the dark matter, which contributes about 25\% [1] to the energy density of the unverse. This also indicates the necessity of supplementing to the existing theory with newer particles having $\mathrm{GeV}$ order mass and very weak interactions.

In this work, we present a new scenario for a naturally tiny Dirac neutrino mass, which accounts for the dark matter and accommodate the leptogeneis. In our model, the small neutrino mass is elegantly induced through a radiative diagram. The new fields responsible for the neutrino mass generation also accommodate the $\mathrm{CP}$ violation and out-of-equilibrium decays to realize the leptogenesis as well as the candidates for the cold dark matter.

Our model: We extend the $S U(3)_{c} \times S U(2)_{L} \times$ $U(1)_{Y}$ SM by introducing two complex scalars: $\chi(\mathbf{1}, \mathbf{1}, 0), \eta(\mathbf{1}, \mathbf{2},-1)$, one real scalar: $\sigma(\mathbf{1}, \mathbf{1}, 0)$, three fermions: $S_{L, R}(\mathbf{1}, \mathbf{1}, 0)$ and three right-handed neutrinos: $\nu_{R}(\mathbf{1}, \mathbf{1}, 0)$. Among these new fields, we appoint the lepton number of the SM leptons for $\nu_{R}$ and $S_{L, R}^{c}$, furthermore, we impose a $U(1)_{D}$ gauge symmetry, under which $\chi, S_{R}$ and $\nu_{R}^{c}$ carry the quantum number 1 , and a $Z_{2}$ discrete symmetry, under which $\eta, \sigma$ and $S_{L, R}$ are odd. Our model exactly conserves the lepton number as well as the $U(1)_{D}$ and $Z_{2}$, so the allowed interactions involving $S_{L, R}$ and $\nu_{R}$ are given by

$$
\mathcal{L} \supset-y \overline{\psi_{L}} \eta S_{L}^{c}-h \sigma \overline{\nu_{R}} S_{R}^{c}-f \chi \overline{S_{R}} S_{L}+\text { h.c. },
$$

*Electronic address: pgu@ictp.it

†Electronic address: utpal@prl.res.in where $\psi_{L}(\mathbf{1}, \mathbf{2},-1)$ denotes the SM left-handed leptons. We also write down the general scalar potential,

$$
\begin{aligned}
V= & m_{\chi}^{2} \chi^{\dagger} \chi+m_{\phi}^{2} \phi^{\dagger} \phi+m_{\eta}^{2} \eta^{\dagger} \eta+\frac{1}{2} m_{\sigma}^{2} \sigma^{2} \\
& +\lambda_{\chi}\left(\chi^{\dagger} \chi\right)^{2}+\lambda_{\phi}\left(\phi^{\dagger} \phi\right)^{2}+\lambda_{\eta}\left(\eta^{\dagger} \eta\right)^{2}+\frac{1}{4} \lambda_{\sigma} \sigma^{4} \\
& +\lambda_{\chi \phi} \chi^{\dagger} \chi \phi^{\dagger} \phi+\lambda_{\chi \eta} \chi^{\dagger} \chi \eta^{\dagger} \eta+\frac{1}{2} \lambda_{\chi \sigma} \chi^{\dagger} \chi \sigma^{2} \\
& +\lambda_{\phi \eta} \phi^{\dagger} \phi \eta^{\dagger} \eta+\lambda_{\phi \eta}^{\prime} \phi^{\dagger} \eta \eta^{\dagger} \phi+\frac{1}{2} \lambda_{\phi \sigma} \phi^{\dagger} \phi \sigma^{2} \\
& +\lambda_{\eta \sigma} \eta^{\dagger} \eta \sigma^{2}+\left[\kappa\left(\phi^{\dagger} \eta\right)^{2}+\frac{1}{\sqrt{2}} \mu \sigma \eta^{\dagger} \phi+\text { h.c. }\right]
\end{aligned}
$$

Here $\phi(\mathbf{1}, \mathbf{2},-1)$ is the SM Higgs. In the following, we will choose the Yukawa coupling $f$ in (1D) to be real and diagonal while the quartic coupling $\kappa$ and the cubic coupling $\mu$ in (2) to be real for convenience but without loss of generality.

The $Z_{2}$ symmetry is unbroken at all energies and hence $\eta$ and $\sigma$ are protected from any nonzero vacuum expectation values (vevs). The gauge symmetry $U(1)_{D}$ is expected to break by $\langle\chi\rangle=\mathcal{O}\left(10^{9} \mathrm{GeV}\right)$. In consequence, the corresponding gauge boson $Z^{\prime}$ obtain its mass $M_{Z^{\prime}}=\sqrt{2} g^{\prime}\langle\chi\rangle$ with $g^{\prime}$ being the $U(1)$ gauge coupling, meanwhile, $S_{L, R}$ realize their Dirac masses $M_{S}=f\langle\chi\rangle$ of the order of $10^{7-8} \mathrm{GeV}$. Subsequently, the electroweak symmetry is broken by $\langle\phi\rangle \simeq 174 \mathrm{GeV}$.

Neutrino mass: As shown in Fig. 1, the neutrinos can get a Dirac mass through the one-loop diagram after the $U(1)_{D}$ and electroweak symmetry breaking. For demonstration, we define $\eta^{0} \equiv \frac{1}{\sqrt{2}}\left(\eta_{R}^{0}+i \eta_{I}^{0}\right)$, where $\eta^{0}$ is the neutral component of $\eta$, and then have

$$
\mathcal{L} \supset-\frac{1}{2}\left(\begin{array}{lll}
\eta_{I}^{0} & \eta_{R}^{0} & \sigma
\end{array}\right)\left(\begin{array}{ccc}
M_{\eta_{I}^{0}}^{2} & 0 & 0 \\
0 & M_{\eta_{R}^{0}}^{2} & \Delta^{2} \\
0 & \Delta^{2} & M_{\sigma}^{2}
\end{array}\right)\left(\begin{array}{c}
\eta_{I}^{0} \\
\eta_{R}^{0} \\
\sigma
\end{array}\right)
$$




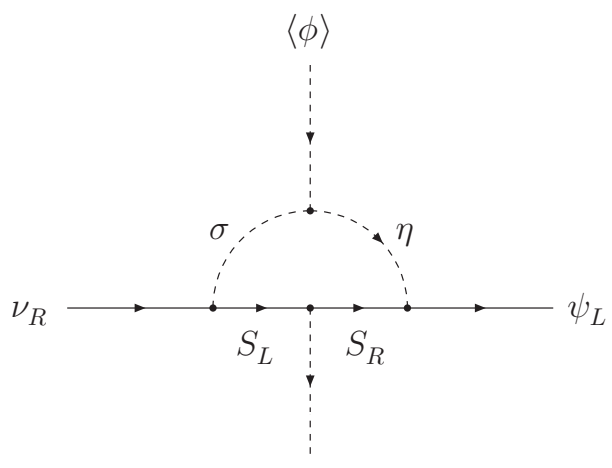

$\langle\chi\rangle$

FIG. 1: The one-loop diagram for generating the radiative neutrino masses.

where

$$
\begin{aligned}
M_{\eta_{I}^{0}}^{2} & \equiv m_{\eta}^{2}+\lambda_{\chi \eta}\langle\chi\rangle^{2}+\left(\lambda_{\phi \eta}+\lambda_{\phi \eta}^{\prime}-\kappa\right)\langle\phi\rangle^{2},( \\
M_{\eta_{R}^{0}}^{2} & \equiv m_{\eta}^{2}+\lambda_{\chi \eta}\langle\chi\rangle^{2}+\left(\lambda_{\phi \eta}+\lambda_{\phi \eta}^{\prime}+\kappa\right)\langle\phi\rangle^{2},(5) \\
M_{\sigma}^{2} & \equiv m_{\sigma}^{2}+\lambda_{\chi \sigma}\langle\chi\rangle^{2}+\lambda_{\phi \sigma}\langle\phi\rangle^{2} \\
\Delta^{2} & \equiv \mu\langle\phi\rangle
\end{aligned}
$$

In the following, we will take $\kappa>0$ and then $M_{\eta_{I}^{0}}<M_{\eta_{R}^{0}}$ for illustration. There is a simple transformation of $\sigma$ and $\eta_{R}^{0}$ to the mass eigenstates $\xi_{1}$ and $\xi_{2}$,

$$
\left(\begin{array}{c}
\eta_{R}^{0} \\
\sigma
\end{array}\right)=\left(\begin{array}{cc}
\cos \vartheta & \sin \vartheta \\
-\sin \vartheta & \cos \vartheta
\end{array}\right)\left(\begin{array}{l}
\xi_{1} \\
\xi_{2}
\end{array}\right)
$$

where the mixing angle $\vartheta$ is

$$
\tan 2 \vartheta=\frac{2 \Delta^{2}}{M_{\sigma}^{2}-M_{\eta_{R}^{0}}^{2}}
$$

The masses of the eigenstates are

$$
\begin{aligned}
& M_{\xi_{1}}^{2}=\frac{1}{2}\left[M_{\eta_{R}^{0}}^{2}+M_{\sigma}^{2}-\sqrt{\left(M_{\eta_{R}^{0}}^{2}-M_{\sigma}^{2}\right)^{2}+4 \Delta^{4}}\right] \\
& M_{\xi_{2}}^{2}=\frac{1}{2}\left[M_{\eta_{R}^{0}}^{2}+M_{\sigma}^{2}+\sqrt{\left(M_{\eta_{R}^{0}}^{2}-M_{\sigma}^{2}\right)^{2}+4 \Delta^{4}}\right]
\end{aligned}
$$

We then give the formula of the radiative neutrino masses,

$$
\begin{aligned}
\left(m_{\nu}\right)_{i j}= & \frac{\sin 2 \vartheta}{32 \pi^{2}} \sum_{k} y_{i k} M_{S_{k}}\left[\frac{M_{\xi_{2}}^{2}}{M_{\xi_{2}}^{2}-M_{S_{k}}^{2}} \ln \left(\frac{M_{\xi_{2}}^{2}}{M_{S_{k}}^{2}}\right)\right. \\
& \left.-\frac{M_{\xi_{1}}^{2}}{M_{\xi_{1}}^{2}-M_{S_{k}}^{2}} \ln \left(\frac{M_{\xi_{1}}^{2}}{M_{S_{k}}^{2}}\right)\right] h_{k j}^{\dagger} .
\end{aligned}
$$

For $M_{S_{k}} \gg M_{\xi_{1,2}}$, we can simplify the above mass matrix as

$$
\left(m_{\nu}\right)_{i j} \simeq \frac{\sin 2 \vartheta}{32 \pi^{2}} M_{\xi_{1}}^{2} \sum_{k} F\left(\frac{M_{\xi_{2}}^{2}}{M_{\xi_{1}}^{2}}, \frac{M_{S_{k}}^{2}}{M_{\xi_{1}}^{2}}\right) \frac{y_{i k} h_{k j}^{\dagger}}{M_{S_{k}}}
$$

with the definition

$$
F(x, y) \equiv x \ln \left(\frac{y}{x}\right)-\ln y .
$$

The function $F\left(\frac{M_{\xi_{2}}^{2}}{M_{\xi_{1}}^{2}}, \frac{M_{S_{k}}^{2}}{M_{\xi_{1}}^{2}}\right)$ could be simply looked on as a constant $c$ if the three $M_{S_{k}}$ are chosen within a few order of magnitude and then the neutrino mass can be written in the simple form,

$$
m_{\nu} \simeq c \frac{\sin 2 \vartheta}{32 \pi^{2}} M_{\xi_{1}}^{2} y \frac{1}{M_{S}} h^{\dagger}
$$

For the purpose of numerical estimation, we input $\left(M_{\eta_{R}^{0}}, M_{\sigma},|\Delta|\right)=$ (300 Gev, $80 \mathrm{Gev}, 30 \mathrm{Gev})$ or $(80 \mathrm{Gev}, 300 \mathrm{Gev}, 30 \mathrm{Gev})$ and then obtain $\left(\tan 2 \vartheta, M_{\xi_{1}}, M_{\xi_{2}}\right)$ = $(\mp 0.02,80 \mathrm{GeV}, 300 \mathrm{GeV})$. Subsequently, we take $M_{S_{k}} \simeq 10^{7} \mathrm{GeV}$ and then derive $c \simeq 270$. For $y \sim h \sim \mathcal{O}\left(10^{-3}\right)$, the neutrino mass comes out to be of the order of $\mathcal{O}(0.01-0.1 \mathrm{eV})$, which is consistent with the neutrino oscillation data and cosmological observations.

Dark matter: It is natural to consider the lighter one between $\xi_{1}$ and $\eta_{I}^{0}$ as the candidate for the dark matter since it has not any decay modes. We first consider that $\xi_{1}$ and $\xi_{2}$ are dominated by $\sigma$ and $\eta_{R}^{0}$, respectively and $\sigma$ is lighter than $\eta_{I}^{0}$. In this case, $\sigma$ is definitely the darkon field [11] that can realize the right amount of the relic density of the cold dark matter when its mass $M_{\sigma}$ is less than $100 \mathrm{GeV}$ and its quartic coupling to the SM Higgs, i.e. $\lambda_{\phi \sigma}$ in the scalar potential (2) is of the order of $\mathcal{O}(0.1)$. Now we check the other possibility that $\eta_{I}^{0}$ is the dark matter. Note that the direct detection of halo dark matter places a limit on the mass degeneracy between $\eta_{R}^{0}$ and $\eta_{I}^{0}$, because the difference must be sufficient to kinematically suppress the scattering of $\eta_{R, I}^{0}$ on nuclei via the tree-level exchange of the $Z$ boson. It has been studied [12] that if $\eta_{I}^{0}$ is expected to be the dark matter, the mass spectrum of mass-eigenstates $\eta_{R, I}^{0}$ should be:

$$
M_{\eta_{R}^{0}}-M_{\eta_{I}^{0}} \simeq(8-9) \mathrm{GeV}
$$

for

$$
M_{\eta_{I}^{0}}=(60-73) \mathrm{GeV}
$$

or

$$
M_{\eta_{R}^{0}}-M_{\eta_{I}^{0}} \simeq(9-12) \mathrm{GeV}
$$




$$
M_{\eta_{I}^{0}}=(73-75) \mathrm{GeV} .
$$

In our model, we have the flexibility to choose the quartic coupling $\kappa$ and other parameters in the scalar potential (2) and then obtain the desired mass spectrum of $\eta_{R, I}^{0}$.

Leptogenesis: Obviously, no lepton asymmetry can be generated in our model because the lepton number is exactly conserved. However, since the sphaleron [13] only have a direct action on the left-handed quarks and leptons, a nonzero lepton asymmetry stored in the lefthanded leptons, which is equal but opposite to that stored in the other fields, can be partially converted to the baryon asymmetry as long as the interactions between the left-handed leptons and the other fields with lepton number are too weak to realize an equilibrium before the electroweak phase transition. For all the SM species, the Yukawa interactions are sufficiently strong to rapidly cancel the left- and right-handed lepton asymmetry. But the effective Yukawa interactions of the ultralight Dirac neutrinos are exceedingly weak and thus will not reach equilibrium until the temperatures fall well below the weak scale. This new type of leptogenesis mechanism is called neutrinogenesis [10].

In our model, the heavy Dirac fermions $S=S_{L}+S_{R}$ have two decay modes as shown in Fig. 2. We calculate the decay width at tree level,

$$
\begin{aligned}
& \Gamma\left(S_{i}^{c} \rightarrow \psi_{L}+\eta^{*}\right)=\Gamma\left(S_{i} \rightarrow \psi_{L}^{c}+\eta\right) \\
= & \frac{1}{16 \pi}\left(y^{\dagger} y\right)_{i i} M_{S_{i}}, \\
& \Gamma\left(S_{i}^{c} \rightarrow \nu_{R}+\sigma\right)=\Gamma\left(S_{i} \rightarrow \nu_{R}^{c}+\sigma\right) \\
= & \frac{1}{32 \pi}\left(h^{\dagger} h\right)_{i i} M_{S_{i}} .
\end{aligned}
$$

At one-loop order as shown in Fig. 3, we compute the $\mathrm{CP}$ asymmetry

$$
\begin{aligned}
\varepsilon_{S_{i}} \equiv & \frac{\Gamma\left(S_{i}^{c} \rightarrow \psi_{L}+\eta^{*}\right)-\Gamma\left(S_{i} \rightarrow \psi_{L}^{c}+\eta\right)}{\Gamma_{S_{i}}} \\
= & \frac{1}{8 \pi} \frac{1}{\left(y^{\dagger} y\right)_{i i}+\frac{1}{2}\left(h^{\dagger} h\right)_{i i}} \\
& \times \sum_{j \neq i} \operatorname{Im}\left[\left(y^{\dagger} y\right)_{i j}\left(h^{\dagger} h\right)_{j i}\right] \frac{M_{S_{i}} M_{S_{j}}}{M_{S_{i}}^{2}-M_{S_{j}}^{2}}
\end{aligned}
$$

Here the total decay width $\Gamma_{S_{i}}$ is given by

$$
\Gamma_{S_{i}}=\frac{1}{16 \pi}\left[\left(y^{\dagger} y\right)_{i i}+\frac{1}{2}\left(h^{\dagger} h\right)_{i i}\right] M_{S_{i}} .
$$

For illustration, we consider the limiting case with $M_{S_{1}} \ll M_{S_{2,3}}$, where the final lepton asymmetry should mainly come from the contributions of the decays of $S_{1}$.
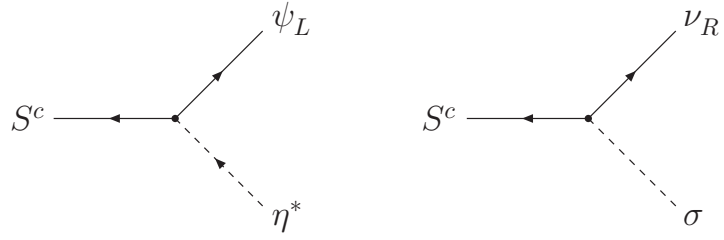

FIG. 2: The heavy Dirac fermions decay to the left-handed leptons and the right-handed neutrinos.

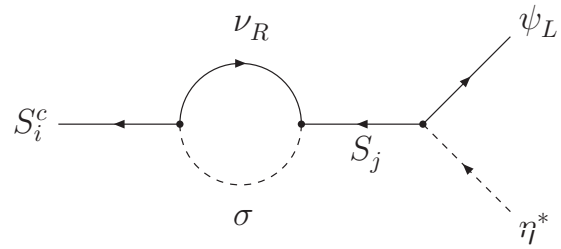

FIG. 3: The heavy Dirac fermions decay to the left-handed leptons at one-loop order.

We can simplify the CP asymmetry (23) as

$$
\begin{aligned}
\varepsilon_{S_{1}} \simeq & -\frac{1}{8 \pi} \frac{1}{\left(y^{\dagger} y\right)_{11}+\frac{1}{2}\left(h^{\dagger} h\right)_{11}} \\
& \times \sum_{j \neq 1} \frac{M_{S_{1}}}{M_{S_{j}}} \operatorname{Im}\left[\left(y^{\dagger} y\right)_{1 j}\left(h^{\dagger} h\right)_{j 1}\right] .
\end{aligned}
$$

Furthermore, we take a simple assumption,

$$
h=y^{*},
$$

and then approach

$$
\varepsilon_{S_{1}} \simeq-\frac{1}{8 \pi} \frac{1}{\left(y^{\dagger} y\right)_{11}} \sum_{j \neq 1} \frac{M_{S_{1}}}{M_{S_{j}}}\left\{\operatorname{Im}\left[\left(y^{\dagger} y\right)_{1 j}^{2}\right]\right\}
$$

Similar to the DI bound [14], we can also deduce an upper bound on the above CP asymmetry by inserting the assumption (26) to the mass formula (13),

$$
\left|\varepsilon_{S_{1}}\right| \leq \frac{4 \pi}{c \sin 2 \vartheta} \frac{M_{S_{1}} m_{3}}{M_{\xi_{1}}^{2}}|\sin \delta|
$$


with $m_{3}$ and $\delta$ being the biggest eigenvalue of the neutrino mass matrix and the $\mathrm{CP}$ phase, respectively. Here we have assumed the neutrinos to be hierarchical [15]. Inputting $M_{S_{1}}=10^{7} \mathrm{GeV}, m_{3}=0.05 \mathrm{eV}, M_{\xi_{1}}=80 \mathrm{GeV}$, $c=270, \sin 2 \vartheta \simeq \tan 2 \vartheta=0.02$ and $\sin \delta=-1$, we derive $\varepsilon_{S_{1}} \simeq-1.8 \times 10^{-7}$ and then obtain the final baryon asymmetry,

$$
\begin{aligned}
\frac{n_{B}}{s} & =\frac{28}{79} \frac{n_{B-L_{S M}}}{s}=-\frac{28}{79} \frac{n_{L_{S M}}}{s} \\
& \simeq-\left.\frac{28}{79} \varepsilon_{S_{1}} \frac{n_{S_{1}}^{e q}}{s}\right|_{T=M_{S_{1}}} \simeq-\frac{1}{15} \frac{\varepsilon_{S_{1}}}{g_{*}} \simeq 10^{-10}
\end{aligned}
$$

as desired to explain the matter-antimatter asymmetry of the universe. Here we have adopted the relativistic degrees of freedom $g_{*}=\mathcal{O}(100)$ [16].

Note when generating the desired baryon asymmetry (29), the decays of $S_{1}$ should satisfy the condition of departure from equilibrium, which is described by

$$
\left.\Gamma_{S_{1}} \lesssim H(T)\right|_{T=M_{S_{1}}}
$$

where

$$
H(T)=\left(\frac{8 \pi^{3} g_{*}}{90}\right)^{\frac{1}{2}} \frac{T^{2}}{M_{\mathrm{Pl}}}
$$

is the Hubble constant with the Planck mass $M_{\mathrm{Pl}} \simeq$ $10^{19} \mathrm{GeV}$. With Eqs. (24), (26), (30) and (31), it is straightforward to perform the condition

$$
\left(y^{\dagger} y\right)_{11} \lesssim\left(\frac{2^{10} \cdot \pi^{5} \cdot g_{*}}{5 \cdot 3^{4}}\right)^{\frac{1}{2}} \frac{M_{S_{1}}}{M_{\mathrm{Pl}}} \sim 10^{-10}
$$

for $M_{S_{1}}=10^{7} \mathrm{GeV}$.

Summary: In this paper, we extended the SM with the requirement of the symmetry that forbids the usual Dirac and Majorana masses of the neutrinos. Through a radiative diagram, the neutrinos obtain tiny Dirac masses suppressed by the heavy new fermions. These fermions also generate a lepton asymmetry stored in the left-handed leptons via their CP-violation and out-of-equilibrium decays. The sphaleron action then partially transfers this lepton asymmetry to a baryon asymmetry so that the observed matter-antimatter asymmetry of the universe can be naturally explained. Moreover, the scalars contributing to the neutrino mass-generation provide consistent candidates for the cold dark matter.
[1] Particle Data Group, W.M. Yao et al., Journal of Physics G 33, 1 (2006); and references therein.

[2] P. Minkowski, Phys. Lett. B 67, 421 (1977); T. Yanagida, in Proc. of the Workshop on Unified Theory and the Baryon Number of the Universe, ed. O. Sawada and A. Sugamoto (KEK, Tsukuba, 1979), p. 95; M. Gell-Mann, P. Ramond, and R. Slansky, in Supergravity, ed. F. van Nieuwenhuizen and D. Freedman (North Holland, Amsterdam, 1979), p. 315; S.L. Glashow, in Quarks and Leptons, ed. M. Lévy et al. (Plenum, New York, 1980), p. 707; R.N. Mohapatra and G. Senjanović, Phys. Rev. Lett. 44, 912 (1980); J. Schechter and J.W.F. Valle, Phys. Rev. D 22, 2227 (1980).

[3] M. Roncadelli and D. Wyler, Phys. Lett. B 133, 325 (1983); P. Roy and O. Shanker, Phys. Rev. Lett. 52, 713 (1984).

[4] P.H. Gu and H.J. He, JCAP 0612, 010 (2006); P.H. Gu, H.J. He, and U. Sarkar, JCAP 0711, 016 (2007); P.H. $\mathrm{Gu}$, H.J. He, and U. Sarkar, Phys. Lett. B 659, 634 (2008); P.H. Gu, Phys. Lett. B 661, 290 (2008); P.H. $\mathrm{Gu}$ and U. Sarkar, arXiv:0712.2793 [hep-ph].

[5] M. Fukugita and T. Yanagida, Phys. Lett. B 174, 45 (1986).

[6] P. Langacker, R.D. Peccei, and T. Yanagida, Mod. Phys. Lett. A 1, 541 (1986); M.A. Luty, Phys. Rev. D 45, 455 (1992); R.N. Mohapatra and X. Zhang, Phys. Rev. D 46, 5331 (1992).
[7] M. Flanz, E.A. Paschos, and U. Sarkar, Phys. Lett. B 345, 248 (1995); M. Flanz, E.A. Paschos, U. Sarkar, and J. Weiss, Phys. Lett. B 389, 693 (1996).

[8] A. Pilaftsis, Phys. Rev. D 56, 5431 (1997).

[9] E. Ma and U. Sarkar, Phys. Rev. Lett. 80, 5716 (1998).

[10] K. Dick, M. Lindner, M. Ratz, and D. Wright, Phys. Rev. Lett. 84, 4039 (2000). For an early related work, see E.Kh. Akhmedov, V.A. Rubakov, and A.Yu. Smirnov, Phys. Rev. Lett. 81, 1359 (1998).

[11] V. Silveira and A. Zee, Phys. Lett. B 161, 136 (1985); J. McDonald, Phys. Rev. D 50, 3637 (1994); C.P. Burgess, M. Pospelov, T. ter Veldhuis, Nucl. Phys. B 619, 709 (2001); X.G. He, T. Li, X.Q. Li, and H.C. Tsai, Mod. Phys. Lett. A 22, 2121 (2007).

[12] R. Barbieri, L.J. Hall, and V.S. Rychkov, Phys. Rev. D 74, 015007 (2006).

[13] V.A. Kuzmin, V.A. Rubakov, and M.E. Shaposhnikov, Phys. Lett. B 155, 36 (1985).

[14] S. Davidson and A. Ibarra, Phys. Lett. B 535, 25 (2002); W. Buchmüller, P. Di Bari, and M. Plümacher, Nucl. Phys. B 665, 445 (2003).

[15] A. Strumia and F. Vissani, hep-ph/0606054; and references therein.

[16] E.W. Kolb and M.S. Turner, The Early Universe, Addison-Wesley, Reading, MA, 1990. 